\title{
Collisional loss of one-dimensional fermions near a $p$-wave Feshbach resonance
}

\author{
Ya-Ting Chang, ${ }^{1}$ Ruwan Senaratne, ${ }^{1}$ Danyel Cavazos-Cavazos, ${ }^{1}$ and Randall G. Hulet ${ }^{1, *}$ \\ ${ }^{1}$ Department of Physics and Astronomy, Rice University, Houston, Texas 77005, USA
}

(Dated: September 23, 2020)

\begin{abstract}
We study collisional loss of a quasi-one-dimensional (1D) spin-polarized Fermi gas near a $p$-wave Feshbach resonance in ultracold ${ }^{6} \mathrm{Li}$ atoms. We measure the location of the $p$-wave resonance in quasi-1D and observe a confinement-induced shift and broadening. We find that the three-body loss coefficient $L_{3}$ as a function of the quasi-1D confinement has little dependence on confinement strength. We also analyze the atom loss with a two-step cascade three-body loss model in which weakly bound dimers are formed prior to their loss arising from atom-dimer collisions. Our data are consistent with this model. We also find a possible suppression in the rate of dimer relaxation with strong quasi-1D confinement. We discuss the implications of these measurements for observing $p$-wave pairing in quasi-1D.
\end{abstract}

The realization of ultracold atomic Fermi gases has provided experimental access to a wide array of phenomena, largely because of the presence of Feshbach resonances (FRs) that provide for externally tunable interactions [14]. In addition to the usual $s$-wave interactions between distinguishable fermions, higher partial-wave interactions may be tuned via FRs [5]. $p$-Wave interactions are of particular interest as they are the dominant low-energy scattering process between identical fermions and are predicted to exhibit phenomena distinct from those observed in $s$-wave interacting Fermi gases [6]. In particular, pairing between identical fermions is an essential ingredient of the Kitaev chain Hamiltonian [7], which supports Majorana zero-modes at the ends of the chain. These zeromodes have been observed in semiconducting nanowires [8], and are a promising candidate platform for faulttolerant quantum computing $[9,10]$.

$p$-Wave FRs have been observed in ${ }^{40} \mathrm{~K}[11-13]$ and ${ }^{6} \mathrm{Li}$ [14-19]. The severe atom losses associated with these resonances, however, have limited their usefulness. Threebody losses, which are suppressed by symmetry in the case of a fermionic two-spin system with $s$-wave interactions [20], are not suppressed for $p$-wave interactions. Much work has been done in characterizing the atom loss associated with $p$-wave FRs [21-24], and there is renewed interest in studying these resonances in reduced dimensions. Recent theoretical work has suggested that threebody losses may be suppressed in quasi-1D [25]. The absence of a centrifugal barrier in 1D results in Feshbach dimers that have extended wavefunctions which overlap less with deeply-bound molecules. If three-body loss is suppressed by this mechanism, it might open a path towards realizing $p$-wave pairing in quasi-1D and emulating the Kitaev chain Hamiltonian.

We present an experimental study of three-body losses near a $p$-wave $\mathrm{FR}$ of identical ${ }^{6} \mathrm{Li}$ fermions in quasi-1D. We measure the three-body loss coefficient $\left(L_{3}\right)$ as a function of 1D confinement for a direct three-body process. We also analyze the observed atom loss within the framework of a cascade model with explicit dimer formation and relaxation steps $[26,27]$, using in situ imaging to reduce the effect of the inhomogeneous density. Finally, we characterize the confinement-induced shifts in the resonance position that appear in quasi-1D [28-32]. These shifts allow us to extract a value for the effective range.

The apparatus and the experimental methods we use to prepare degenerate Fermi gases have been described previously [33-35]. A ${ }^{6} \mathrm{Li}$ degenerate Fermi gas is first prepared in the two lowest hyperfine states of the $S_{1 / 2}$ manifold (states $|1\rangle$ and $|2\rangle$, respectively) at $595 \mathrm{G}$, and then loaded into a crossed-beam dipole trap formed by three linearly-polarized mutually-orthogonal laser beams of wavelength $\lambda=1.064 \mu \mathrm{m}$. Each beam is retroreflected, with the polarizations of the incoming and retro-reflected beams initially set to be perpendicular to each other to avoid lattice formation. We eliminate state $|1\rangle$ from the trap with a resonant burst of light. At this stage, we obtain $9(1) \times 10^{4}$ atoms in state $|2\rangle$ in a nearly isotropic harmonic trap with a geometric-mean trapping frequency of $2 \pi \times 305(2) \mathrm{Hz}$, and at a temperature $T / T_{F} \approx$ 0.1 where, $T_{F}$ is the Fermi temperature. The optical trap depths are increased and the polarizations of the retroreflected beams are rotated to achieve a $7 E_{r}$ deep 3D optical lattice, where $E_{r}=h^{2} /\left(2 m \lambda^{2}\right)=k_{\mathrm{B}} \times 1.41 \mu \mathrm{K}$ is the recoil energy, and $m$ is the atomic mass. During the lattice ramp-up, a co-propagating beam of 532 $\mathrm{nm}$ light is introduced along each trapping-beam dimension to flatten the trapping potential [33, 34]. By tuning these compensation beam powers, we create a $3 \mathrm{D}$ band insulator with a central density of approximately 1 atom 
per site. In order to produce a 2D lattice, which is an array of quasi-1D tubes, we slowly turn off the compensation beams and the vertical lattice beam, while increasing the intensity of the two remaining beams to achieve a desired 2D lattice depth, $V_{L}$. This depth determines the confinement in the quasi-1D traps, which is parameterized by $a_{\perp}=\sqrt{2 \hbar / m \omega_{r}}$ transversely and $R_{F}$ axially, where $\omega_{r}=\sqrt{4 E_{r} V_{L}} / \hbar$ is the trapping frequency of a lattice site when approximated as a harmonic potential, and $R_{F}\left(N_{t, j}, \omega_{z}\right)=\sqrt{\left(2 N_{t, j}+1\right) \hbar / m \omega_{z}}$ is the Fermi radius of tube $j$ with number of atoms $N_{t, j}$ and an axial frequency $\omega_{z}$. The aspect ratio of the quasi-1D tubes, $\omega_{r} / \omega_{z} \approx 170$. We load a maximum of around 30 atoms per quasi-1D tube with $T<T_{F}$ to avoid exciting any radial modes.

We use a two-step servo scheme to stabilize the current in the coils producing the Feshbach magnetic field, because the ${ }^{6} \mathrm{Li}|1\rangle-|1\rangle$-wave FR near $159 \mathrm{G}$ is very narrow. The first servo, $S_{1}$, provides the large dynamic range required to run our experimental sequence, while the second servo, $S_{2}$, controls the current in a bypass circuit added in parallel to the magnetic coils. This improves the stability of the magnetic field to $\pm 10 \mathrm{mG}$ and provides finer magnetic-field resolution. After reaching the hold field $B$, the atoms are transferred into $|1\rangle$ with a $\pi$-pulse of duration $75 \mu$ s using RF radiation resonant with the $|1\rangle-|2\rangle$ transition. After a hold time $\tau$, we ramp the field back to $595 \mathrm{G}$, where the distribution of the remaining atoms is imaged using in situ phase-contrast imaging with a probe beam propagating perpendicular to the tube axis [35]. By using the inverse Abel transform, which exploits the approximate cylindrical symmetry of the 2D lattice, we measure the distribution with a spatial resolution of approximately three lattice constants. We sector the $2 \mathrm{D}$ lattice into concentric shells in which the tubes have similar chemical potentials, $\mu$. This procedure is useful as scattering processes are in general energy-dependent, so observables depend on rate coefficients that are averaged over the Fermi-Dirac distribution for atoms in each tube.

We characterize the $|1\rangle-|1\rangle$-wave $\mathrm{FR}$ in $3 \mathrm{D}$ and quasi$1 \mathrm{D}$ by measuring atom loss as functions of $B$ and $\tau$. In 3D, we find the onset of loss at 159.05(1) G, which agrees with previous measurements of the location of this resonance in $3 \mathrm{D}[15,17]$ but differs with other measurements $[19,36]$ by a few 10's of $\mathrm{mG}$. We are not able to resolve the expected doublet feature arising from the dipole-dipole interaction $[12,19,37]$ because of limitations of the field stability. All the $1 \mathrm{D}$ data in this paper were measured with the magnetic field aligned with the z-axis, and thus only involve collisions with the $m_{l}=0$ projection of the angular momentum. As $V_{L}$ is increased, we observe a confinement-
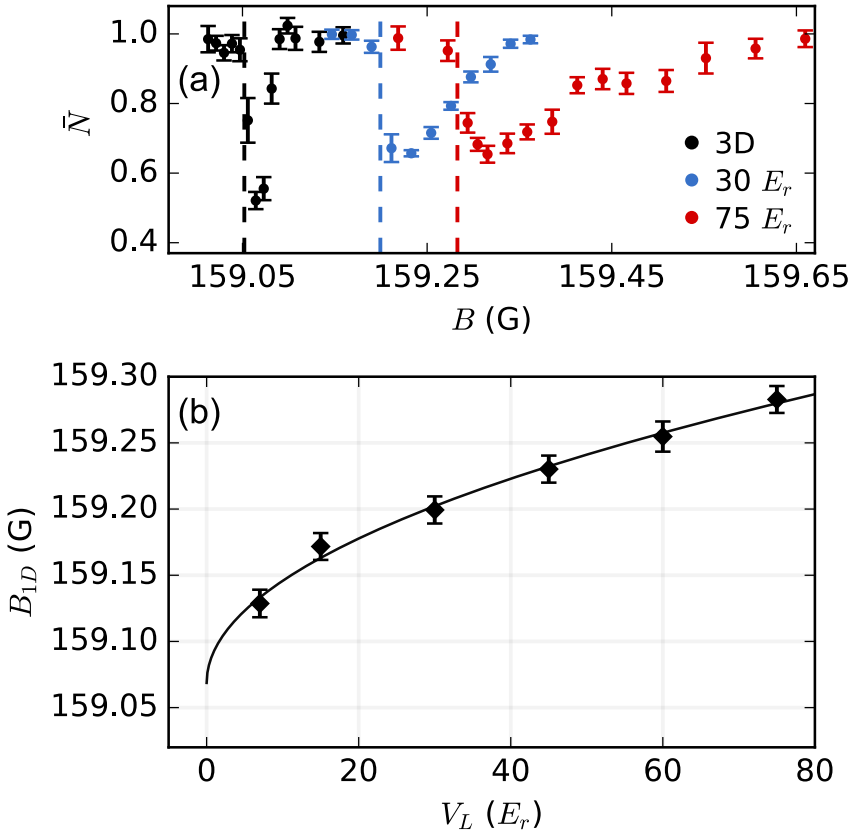

FIG. 1. (a) $p$-Wave resonances in 3D and quasi-1D measured with magnetic-field-dependent loss. Dashed lines show the resonance position for each $V_{L}$. We define the resonance field for zero-momentum collisions, which corresponds to the onset $(15 \%$ loss, to overcome atom number fluctuation) of the observed atomic loss. Data are averaged over 6 experimental runs and error bars are the standard error of the mean. (b) Diamonds show $B_{1 D}$ vs $V_{L}$. The solid curve shows the result of fitting the data to Eq. 3, where the effective range $\alpha_{p}=$ $0.14(1) a_{0}^{-1}$ and $B_{3 D}=159.07(1) \mathrm{G}$ are fitted parameters. Error bars are the statistical uncertainty arising from atom number fluctuation and field instability. In both (a) and (b), $\tau$ is chosen such that peak loss is $30-50 \%$ of total atom number for each value of $V_{L}: 2.5 \mathrm{~ms}$ for $3 \mathrm{D}, 0.5 \mathrm{~ms}$ for $7 E_{r}$ and 0.2 ms for $15-75 E_{r}$.

induced shift in the resonance field and broadening of the atom-loss feature, as shown in Fig. 1(a).

We review $p$-wave scattering in $3 \mathrm{D}$ and quasi-1D to show how the measured confinement-induced shift can be used to extract $\alpha_{p}$, the $3 \mathrm{D}$ effective range. For low-energy collisions in $3 \mathrm{D}$, the cotangent of the phase-shift $\delta_{p}$ associated with $p$-wave scattering can be expanded as a function of scattering volume, $V_{p}$, and effective range, $\alpha_{p}[38]$ :

$$
k^{3} \cot \left(\delta_{p}(k)\right)=-\frac{1}{V_{p}}-\alpha_{p} k^{2}+O\left(k^{4}\right),
$$

where $\alpha_{p}>0$ and has units of inverse length. These 
scattering properties are modified in quasi-1D,

$$
k \cot \left(\delta_{p}(k)\right)=-\frac{1}{l_{p}}-\xi_{p} k^{2}+O\left(k^{4}\right),
$$

where $l_{p}$ is the $1 \mathrm{D}$ scattering length and $\xi_{p}$ is the $1 \mathrm{D}$ effective range, which has units of length. These quasi-1D scattering parameters are given by $l_{p}=$ $3 a_{\perp}\left[a_{\perp}^{3} / 2 V_{p}+\alpha_{p} a_{\perp}+6|\zeta(-1 / 2)|\right]^{-1}$ and $\xi_{p}=\alpha_{p} a_{\perp}^{2} / 6$ [30-32], where $\zeta$ is the Riemann zeta function $(\zeta(-1 / 2) \approx$ $-0.208)$. The second and third terms in $1 / l_{p}$ lead to a confinement-induced shift in the resonance location. In this formalism, only dynamics along the axial dimension are relevant, and scattering quantities, such as the elastic scattering cross-section, are expressed in units appropriate for $1 \mathrm{D}$.

By performing a coupled-channel calculation, which requires detailed knowledge of the inter-atomic potentials [39], we obtain an expansion $1 / V_{p}(B)$ up to second order in $B$. The effective range $\alpha_{p}$ can be approximated as a constant independent of $B$ for the relevant range of magnetic field. The FR in 3D occurs at the magnetic field $B_{3 \mathrm{D}}$ at which $V_{p}$ diverges. Similarly, in quasi-1D, the resonance occurs when $l_{p}$ diverges at a magnetic field $B_{1 \mathrm{D}}$, which is a function of $V_{L}$ and $\alpha_{p}$. The confinementinduced shift, $\delta_{B}\left(V_{L}, \alpha_{p}\right)=B_{1 D}-B_{3 D}$, can be approximated to leading order in confinement strength $V_{L}$ by [40]

$$
\delta_{B}=\frac{-2 m E_{r}}{\left.\hbar^{2} \frac{\partial\left(1 / V_{p}\right)}{\partial B}\right|_{B=B_{3 D}}} \alpha_{p} \sqrt{V_{L}}
$$

We cannot accurately measure $B_{3 D}$ for $m_{l}=0$ alone due to the unresolved $\left|m_{l}\right|=1$ collisions in $3 \mathrm{D}$, so we fit the measured $\delta_{B}$ as a function of $V_{L}$ to Eq. 3 by taking $\alpha_{p}$ and $B_{3 D}$ as fitting parameters. The result of the fit to the quasi-1D data is shown by the solid curve in Fig. 1(b). We obtain $\alpha_{p}=0.14(1) a_{0}^{-1}$ which is consistent with our coupled-channel result of $0.1412 a_{0}^{-1}$, where $a_{0}$ is the Bohr radius, and $B_{3 D}=159.07(1)$ which is consistent with our loss-onset measurement and a dipolar splitting of $10 \mathrm{mG}$ in 3D [19]. We also find a consistent value by analyzing previous measurements performed on a $2 \mathrm{D}$ gas of ${ }^{6} \mathrm{Li}$ in state $|1\rangle[21,40]$.

The observed atom loss is presumably due to the formation of deeply-bound molecules. To characterize the loss, we measured $N$, the number of atoms remaining in the trap after a hold time $\tau$ for various $B$ and $V_{L}$. Background-gas collisions lead to a $1 / e$ atom lifetime of $38 \mathrm{~s}$ in this apparatus, and are negligible for this analysis.
Atom loss due to three-body collisions is described by

$$
\frac{\dot{N}}{N}=-L_{3} n^{2}
$$

where $n^{2}=\left(N_{t, c} / 2 R_{F, c}\right)^{2}$ is the squared atomic line density for a central tube, determined using a length-scale of twice the local Fermi radius $R_{F, c}$. We measure the time evolution with $V_{L}$ between 15 and $75 E_{r}$ and extract $L_{3}$ by fitting loss vs $\tau$ to Eq. 4. Fig. 2(a) shows such a fit to typical loss data. Since $L_{3}$ also depends on $\Delta B$, the field detuning from resonance, we extract $L_{3}$ from the time evolution at several $\Delta B$ to find the peak value for each $V_{L}$. The peak $L_{3}$ for all $V_{L}$ are found to be approxi-

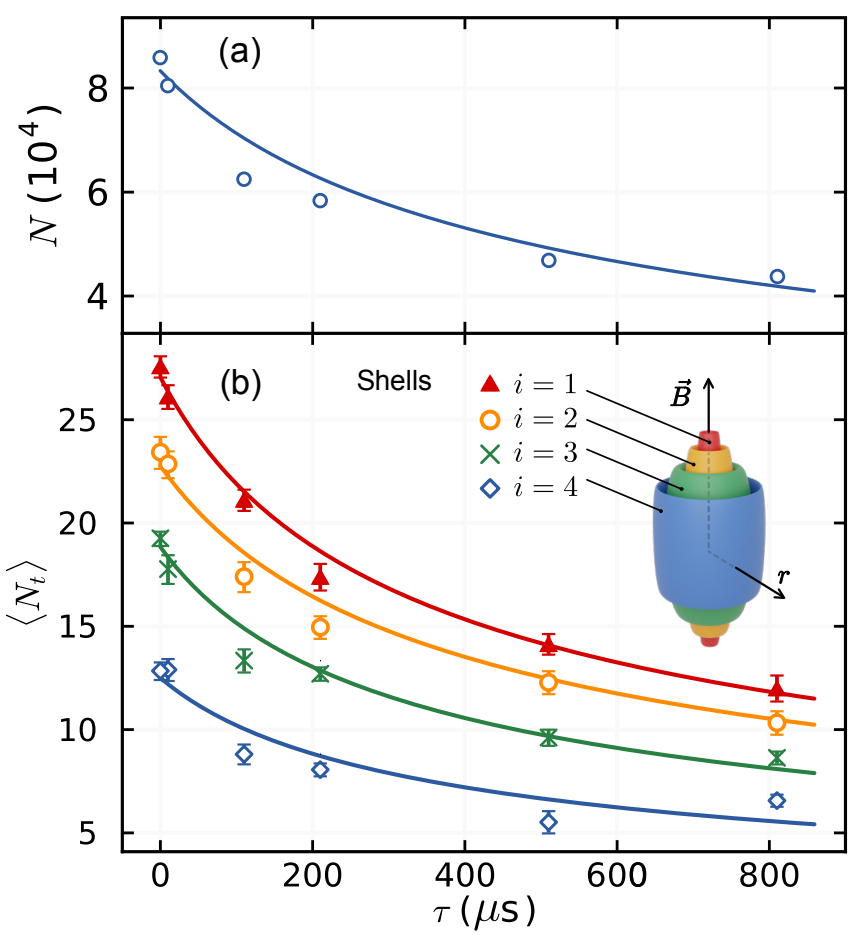

FIG. 2. Typical time evolution of (a) total number in the entire sample and (b) averaged tube population $\left\langle N_{t}\right\rangle$ in 4 shells. For these data, $\Delta B=30 \mathrm{mG}$ and $V_{L}=75 E_{r}$. The different colors and symbols in (b) indicate different shells with approximately uniform initial atom number per tube. The shells are labeled from $i=1$, the inner-most, to $i=4$, the outer-most. Solid curves show fits to Eq. 4 to extract $L_{3}$ with the squared atomic density (a) $n^{2}=\left(N_{t, c} / 2 R_{F, c}\right)^{2}$ of a central tube and (b) $n^{2}=\left(\left\langle N_{t}\right\rangle_{i} / 2 R_{F, i}\right)^{2}$ of a typical tube in each shell. The corresponding $L_{3}$ values are plotted in Fig. 3. Data points are averaged over 5 shots, and the standard error of the mean is (a) approximately equal to the symbol size and (b) indicated by the error bars. 
mately $7(2) \times 10^{-6} \mathrm{~cm}^{2} / \mathrm{s}$. We observe no dependence on $1 \mathrm{D}$ confinement in this range [40]. Due to the inhomogeneity of the initial distribution of atoms across the 2D lattice, however, we find a rather poor agreement of the data to Eq. 4.

The results of a more comprehensive analysis of the same data that provides an improved fit to Eq. 4 is shown in Fig. 2(b). Here, we group the tubes into separate cylindrical shells (labeled by $i=1-4$ ) with an averaged atom number per tube $\left\langle N_{t}\right\rangle_{i}[40]$ and a corresponding Fermi temperature $T_{F, i}$. Figure $3(\mathrm{a})$ shows $L_{3}$ for each shell extracted from data with $V_{L}=75 E_{r}$ vs $\Delta B$. The peak $L_{3}$ for each shell is in the range of $5 \times 10^{-6} \mathrm{~cm}^{2} / \mathrm{s}$ to $1 \times 10^{-5} \mathrm{~cm}^{2} / \mathrm{s}$, and is similar to the peak $L_{3}$ extracted from the whole atomic cloud.

In [25], Zhou and Cui suggest that the rate of threebody loss near a $p$-wave FR can be suppressed by reducing the overlap between the wavefunctions of a deeplybound molecule and a Feshbach dimer with increasing confinement. To investigate this hypothesis, we analyze our observed loss data using a cascade model of two consecutive two-body processes instead of a direct three-body event: two atoms resonantly form a dimer, followed by a collision between the dimer and an atom, resulting in a deeply-bound molecule and an atom [26]. This approach has previously been applied to the particular $p$-wave FR we study, but in $3 \mathrm{D}$ and quasi-2D [27]. It is the natural formalism in which to evaluate the predicted suppression, as it models the formation and relaxation of dimers. The equations governing this loss process are

$$
\begin{aligned}
& \frac{d N_{a}}{d t}=2 \frac{\Gamma}{\hbar} N_{d}-2 K_{a a} \frac{N_{a}\left(N_{a}-1\right)}{4 R_{F}}-K_{a d} \frac{N_{a} N_{d}}{2 R_{F}}, \\
& \frac{d N_{d}}{d t}=-\frac{\Gamma}{\hbar} N_{d}+K_{a a} \frac{N_{a}\left(N_{a}-1\right)}{4 R_{F}}-K_{a d} \frac{N_{a} N_{d}}{2 R_{F}},
\end{aligned}
$$

where $N_{a}$ is the number of atoms, $N_{d}$ is the number of dimers, $K_{a a}$ is the two-body event rate for atom-atom collisions converting atoms into dimers, and $K_{a d}$ is the two-body atom-dimer inelastic collision event rate. $\Gamma$, the one-body decay rate of dimers is the width of the FR. The rate of dimer formation is proportional to the number of possible pairs of atoms, given by $N_{a}\left(N_{a}-1\right) / 2$ !

$K_{a d}$ is of particular interest, as it depends on the overlap between dimers and deeply-bound molecules. Both $\Gamma$ and $K_{a a}$ are related to the elastic scattering cross-section, $\sigma_{1 D}(E)$, which can be calculated, thus constraining the fit to the cascade process to a single parameter, $K_{a d} . \sigma_{1 D}(E)$ may be approximated by a Lorentzian in collision energy,
$E=\hbar^{2} k^{2} / m$, centered at the above-threshold binding energy of the Feshbach dimer $E_{\text {res }}=-\hbar^{2} / l_{p} \xi_{p} m>0$ and with width $\Gamma=\left(\hbar / \xi_{p}\right) \sqrt{4 E_{\text {res }} / m}[6,40]$.

$K_{a a}$ may be calculated by averaging $\sigma_{1 \mathrm{D}}\left(k_{r}\right)$ over the ensemble of pairs of atoms with relative momentum $k_{r}$ and velocity $v_{r}$

$$
K_{a a}=\left\langle\sigma_{1 \mathrm{D}}\left(k_{r}\right) v_{r}\right\rangle=\hbar \int_{-\infty}^{\infty} d k_{r} \sigma_{1 \mathrm{D}}\left(k_{r}\right) v_{r} P\left(k_{r}\right),
$$

where $P\left(k_{r}\right)$ is the probability density function of $k_{r}$ obtained from the density distribution of a trapped Fermi gas [40]. We assume a global temperature $T$ across the entire sample. However, $\mu$ varies significantly from tubeto-tube due to the density inhomogeneity across the $2 \mathrm{D}$ lattice. This effect is mitigated by sectoring the cloud into shells of similar $\mu$, as discussed earlier, thus giving a distinct value of $K_{a a}$ for each shell. For each quasi-1D tube, $\mu$ is determined by $N_{t, j}$ and $T$.

Although we cannot directly measure $T$, we exploit the fact that at a sufficiently large $\Delta B$, the rate equations can be approximated as a direct three-body loss process with a loss coefficient $\tilde{L_{3}}=(3 / 2) \hbar K_{a d} K_{a a} / \Gamma$ under the assumptions of a steady-state dimer population $\left(d N_{d} / d t=0\right)$ and $\Gamma / \hbar \gg K_{a d} N_{a} / 2 R_{F}[27]$. Assuming that these assumptions hold for large $\Delta B$, we fit the measured values of $L_{3}$ for each shell with $T$ and $K_{a d}$ as fitting parameters to $\tilde{L_{3}}$. We find that $T=0.1 T_{F, 1}$, and that $K_{a d}=0.67$ $\mathrm{cm} / \mathrm{s}$ is independent of field for $\Delta B>100 \mathrm{mG}$. The assumptions given above are confirmed in this range. The solid lines in Fig. 3(a) show $\tilde{L_{3}}$ for each shell.

The extracted $K_{a d}$ values from fitting loss data for $V_{L}=$ $75 E_{r}$ to Eqs. 5 using the calculated values of $\Gamma$ and $K_{a a}$ are shown in Fig. 3(b) for the full range of $\Delta B$ [40]. We find that under these conditions, Eqs. 5 model the time behavior of the observed loss as well as Eq. 4. The values of $K_{a d}$ extracted for $\Delta B>50 \mathrm{mG}$ are field independent. The observed field independence strongly supports the cascade model as the atom-dimer collision process is inherently non-resonant. In the dimer formation step, the atoms must collide with a momentum dictated by the binding energy of the dimer, which is field-dependent. The dimer relaxation step, however, may proceed for any collision momentum, as the atom receives the binding energy of the deeply bound molecule.

The behavior of $K_{a d}$ for $\Delta B<50 \mathrm{mG}$ is consistent with a suppression of the rate of dimer relaxation. The spatial overlap of the dimer and deeply-bound wavefunctions increases with $\kappa a_{\perp}$, where $\kappa=\sqrt{m E_{\text {res }}} / \hbar$, so the predicted suppression is strongest for small $\Delta B$, where 


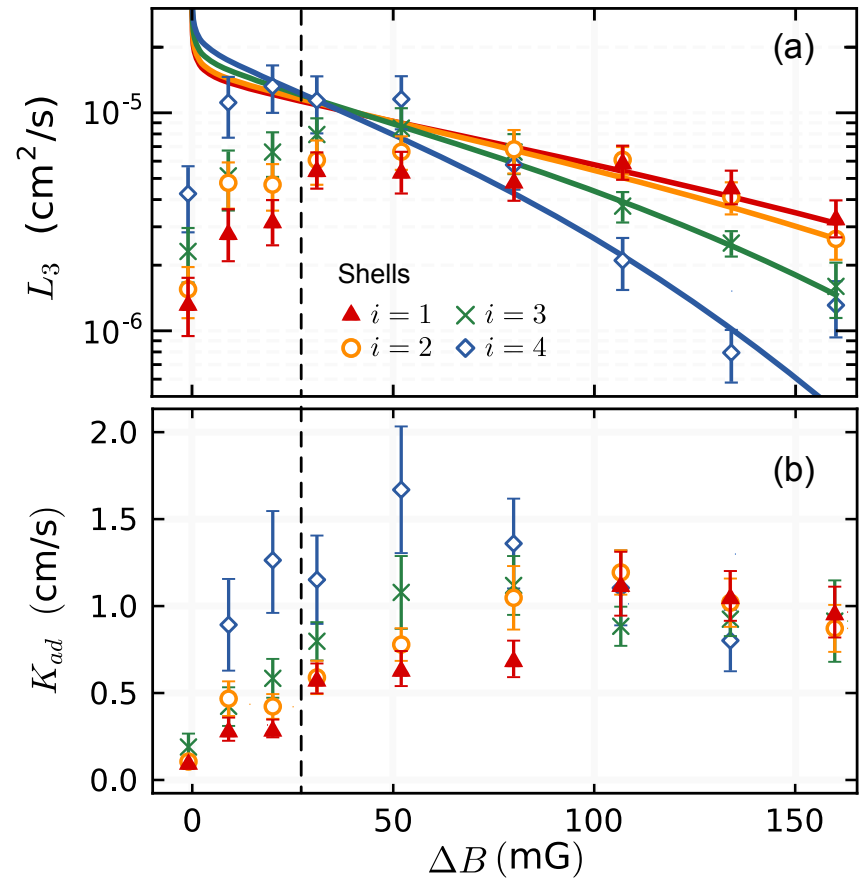

FIG. 3. (a) $L_{3}$ vs $\Delta B$ for $V_{L}=75 E_{r}$. $L_{3}$ is obtained by fitting $N_{t, i}$ vs $\tau$ to Eq. 4 for each shell. An example of this data is given in Fig. 2(b) for $\Delta B=30 \mathrm{mG}$. Solid curves show $(3 / 2) \hbar K_{a d} K_{a a} / \Gamma$ with a constant $K_{a d}=0.67 \mathrm{~cm} / \mathrm{s}$, calculated for $T=0.1 T_{F, 1}$, where $T_{F, 1}=4.8(2) \mu \mathrm{K}$. (b) $K_{a d}$ vs $\Delta B$. $K_{a d}$ is extracted by fitting $\left\langle N_{t}\right\rangle_{i}$ vs $\tau$ to Eq. 5, using the calculated values of $\Gamma$ and $K_{a a}$. Black dashed line indicates $\Delta B=27 \mathrm{mG}$, which corresponds to $\kappa a_{\perp}=1 / 2$ for $V_{L}=75$ $E_{r}$ [25]. Error bars are one-sigma confidence intervals for the fitting parameters $L_{3}$ and $K_{a d}$. The large uncertainty in the fitted values for the outermost shell is indicative of small $N_{t}$.

$E_{\text {res }}$ is smallest. The suppression is expected to be significant for $\kappa a_{\perp}<1 / 2$ [25], which for $V_{L}=75 E_{r}$ corresponds to $\Delta B<27 \mathrm{mG}$. Another interpretation of the small-detuning behavior of $K_{a d}$ is that the cascade model breaks down due to, for example, the existence of a shallow three-body bound state [41].

This work is the first detailed experimental study of $p$ wave collisions in quasi-1D. We confirm the confinementinduced shift and broadening as a function of $V_{L}$. The confinement-induced shift agrees well with quasi-1D theory [32] and the extracted value of $\alpha_{p}$ agrees with previous work [21]. We measure $L_{3}$ as a function of $V_{L}$ and find no dependence up to $75 E_{r}$. The magnetic field independence of $K_{a d}$ for $\Delta B>50 \mathrm{mG}$ confirms the cascade model $[26,27]$ for three-body loss in quasi-1D in the regime of large $\Delta B(>100 \mathrm{mG})$, as well as for intermediate $\Delta B$
(50-100 $\mathrm{mG}$ ) where the cascade model is not well approximated by the three-body loss rate equation.

The suppression in $K_{a d}$ at $\Delta B<50 \mathrm{mG}$ is possibly explained by $p$-wave dimer stretching [25]. Achieving greater suppression in ${ }^{6} \mathrm{Li}$ by increasing $V_{L}$ is challenging since at a fixed $\Delta B, \kappa a_{\perp} \propto 1 / V_{L}^{1 / 4}[40]$, but future work at even higher $V_{L}$ or with improved magnetic field resolution and stability would enable further study of this narrow feature. Our result also provides insight into a potential pathway towards observing pairing between identical fermions in cold atom systems. Suppressing loss in heavier fermions with FRs, such as ${ }^{40} \mathrm{~K}$ [11-13], ${ }^{161}$ Dy [42], and ${ }^{167} \operatorname{Er}$ [43], is promising, as small values of $\kappa a_{\perp}$ may be more readily achieved in these atoms.

Note added. - During the peer-review process, another group reported on a similar experiment [44]. Although both groups observe similar overall atom loss, they report a suppression of $L_{3} \propto V_{L}^{-1}$, while we find $L_{3}$ independent of $V_{L}$ over a wide range (Fig. S2). The difference lies in the choice between defining $L_{3}$ using the $3 \mathrm{D}$ or the $1 \mathrm{D}$ densities. In their analysis, $L_{3}$ is defined in terms of the 3D density of a tube, which increases with $V_{L}^{1 / 2}$, while we use the $1 \mathrm{D}$ line density. While the two results are consistent, we argue that $1 \mathrm{D}$ densities are most appropriate based on physical and practical considerations. Physically, the dimensionless quantity $\kappa a_{\perp}$ parameterizes the effective dimensionality of the system near a FR, and the peak values of $L_{3}$ we report were measured in regions where $\kappa a_{\perp}<1$. Practically, 1D units make it clear that the peak loss rate is independent of $V_{L}$.

We would like to thank T. L. Yang for his contributions to the apparatus and W. I. McAlexander for his coupled-channel code. This work was supported in part by the Army Research Office Multidisciplinary University Research Initiative (Grant Nos. W911NF-14-1-0003 and W911NF-17-1- 0323), the NSF (Grant No. PHY1707992), and the Welch Foundation (Grant No. C-1133). D. C. acknowledges financial support from CONACyT (Mexico, Scholarship No. 472271).

*randy@rice.edu

[1] B. DeMarco and D. S. Jin, Science 285, 1703 (1999).

[2] A. G. Truscott, K. E. Strecker, W. I. McAlexander, G. B. Partridge, and R. G. Hulet, Science 291, 2570 (2001).

[3] F. Schreck, L. Khaykovich, K. L. Corwin, G. Ferrari, T. Bourdel, J. Cubizolles, and C. Salomon, Phys. Rev. Lett. 87, 080403 (2001).

[4] S. R. Granade, M. E. Gehm, K. M. O'Hara, and J. E. 
Thomas, Phys. Rev. Lett. 88, 120405 (2002).

[5] C. Chin, R. Grimm, P. Julienne, and E. Tiesinga, Rev. Mod. Phys. 82, 1225 (2010).

[6] V. Gurarie and L. Radzihovsky, Ann. Phys. (NY) 322, 2 (2007).

[7] A. Y. Kitaev, Phys. Usp. 44, 131 (2000).

[8] S. D. Sarma, M. Freedman, and C. Nayak, Quantum Inf. 1, 15001 (2015).

[9] S. B. Bravyi and A. Y. Kitaev, Ann. Phys. (NY) 298, 210 (2002).

[10] A. Stern and N. H. Lindner, Science 339, 1179 (2013).

[11] C. A. Regal, C. Ticknor, J. L. Bohn, and D. S. Jin, Phys. Rev. Lett. 90, 053201 (2003).

[12] K. Günter, T. Stöferle, H. Moritz, M. Köhl, and T. Esslinger, Phys. Rev. Lett. 95, 230401 (2005).

[13] C. Luciuk, S. Trotzky, S. Smale, Z. Yu, S. Zhang, and J. H. Thywissen, Nat. Phys. 12, 599 (2016).

[14] J. Zhang, E. G. M. van Kempen, T. Bourdel, L. Khaykovich, J. Cubizolles, F. Chevy, M. Teichmann, L. Tarruell, S. J. J. M. F. Kokkelmans, and C. Salomon, Phys. Rev. A 70, 030702 (2004).

[15] C. H. Schunck, M. W. Zwierlein, C. A. Stan, S. M. F. Raupach, W. Ketterle, A. Simoni, E. Tiesinga, C. J. Williams, and P. S. Julienne, Phys. Rev. A 71, 045601 (2005).

[16] Y. Inada, M. Horikoshi, S. Nakajima, M. KuwataGonokami, M. Ueda, and T. Mukaiyama, Phys. Rev. Lett. 101, 100401 (2008).

[17] J. Fuchs, C. Ticknor, P. Dyke, G. Veeravalli, E. Kuhnle, W. Rowlands, P. Hannaford, and C. J. Vale, Phys. Rev. A 77, 053616 (2008).

[18] R. A. W. Maier, C. Marzok, C. Zimmermann, and P. W. Courteille, Phys. Rev. A 81, 064701 (2010).

[19] M. Gerken, B. Tran, S. Häfner, E. Tiemann, B. Zhu, and M. Weidemüller, Phys. Rev. A 100, 050701 (2019).

[20] D. S. Petrov, C. Salomon, and G. V. Shlyapnikov, Phys. Rev. Lett. 93, 090404 (2004).

[21] M. Waseem, Z. Zhang, J. Yoshida, K. Hattori, T. Saito, and T. Mukaiyama, J. Phys. B 49, 204001 (2016).

[22] M. Waseem, T. Saito, J. Yoshida, and T. Mukaiyama, Phys. Rev. A 96, 062704 (2017).

[23] J. Yoshida, T. Saito, M. Waseem, K. Hattori, and T. Mukaiyama, Phys. Rev. Lett. 120, 133401 (2018).

[24] M. Waseem, J. Yoshida, T. Saito, and T. Mukaiyama, Phys. Rev. A 98, 020702 (2018).
[25] L. Zhou and X. Cui, Phys. Rev. A 96, 30701 (2017).

[26] J. Li, J. Liu, L. Luo, and B. Gao, Phys. Rev. Lett. 120, 193402 (2018).

[27] M. Waseem, J. Yoshida, T. Saito, and T. Mukaiyama, Phys. Rev. A 99, 052704 (2019).

[28] M. Olshanii, Phys. Rev. Lett. 81, 938 (1998).

[29] T. Bergeman, M. G. Moore, and M. Olshanii, Phys. Rev. Lett. 91, 163201 (2003).

[30] B. E. Granger and D. Blume, Phys. Rev. Lett. 92, 133202 (2004).

[31] L. Pricoupenko, Phys. Rev. Lett. 100, 170404 (2008).

[32] D. V. Kurlov and G. V. Shlyapnikov, Phys. Rev. A 95, 032710 (2017).

[33] R. A. Hart, P. M. Duarte, T.-L. Yang, X. Liu, T. Paiva, E. Khatami, R. T. Scalettar, N. Trivedi, D. A. Huse, and R. G. Hulet, Nature 519, 211 (2015).

[34] T. L. Yang, P. Grišins, Y. T. Chang, Z. H. Zhao, C. Y. Shih, T. Giamarchi, and R. G. Hulet, Phys. Rev. Lett. 121, 103001 (2018).

[35] R. G. Hulet, J. H. V. Nguyen, and R. Senaratne, Rev. Sci. Instrum. 91, 011101 (2020).

[36] T. Nakasuji, J. Yoshida, and T. Mukaiyama, Phys. Rev. A 88, 12710 (2013).

[37] C. Ticknor, C. A. Regal, D. S. Jin, and J. L. Bohn, Phys. Rev. A 69, 042712 (2004).

[38] C. J. Joachain, Quantum Collision Theory (NorthHolland, 1975)

[39] M. Houbiers, H. T. C. Stoof, W. I. McAlexander, and R. G. Hulet, Phys. Rev. A 57, R1497 (1998).

[40] See Supplemental Material at [URL] for information regarding the derivation of Eq. 3, the confinement-induced shift in quasi-2D, the dependence of $L_{3}$ vs $V_{L}$, in situ imaging data, the fitting of time evolution data to the cascade model, the quasi-1D $p$-wave scattering cross-section, the probability density function of $k_{r}$, and the dependence of $\kappa a_{\perp}$ on $V_{L}$.

[41] M. Schmidt, H.-W. Hammer, and L. Platter, Phys. Rev. A 101, 062702 (2020).

[42] K. Baumann, N. Q. Burdick, M. Lu, and B. L. Lev, Phys. Rev. A 89, 020701 (2014).

[43] S. Baier, D. Petter, J. H. Becher, A. Patscheider, G. Natale, L. Chomaz, M. J. Mark, and F. Ferlaino, Phys. Rev. Lett. 121, 093602 (2018).

[44] A. S. Marcum, F. R. Fonta, A. M. Ismail, and K. M. O'Hara, arXiv:2007.15783 [physics.atom-ph] (2020). 


\title{
Supplementary material for "Collisional loss of one-dimensional fermions near a $p$-wave Feshbach resonance"
}

\author{
Ya-Ting Chang, Ruwan Senaratne, Danyel Cavazos-Cavazos, and Randall G. Hulet* \\ Department of Physics and Astronomy, Rice University, Houston, Texas 77005, USA
}

(Dated: September 23, 2020)

\section{CONFINEMENT-INDUCED SHIFT $\delta_{B}$ IN QUASI-1D}

The Feshbach resonance occurs at a magnetic field $B_{1 D}$ where the $1 \mathrm{D} p$-wave scattering length $l_{p}$ diverges $[1-3]$

$$
\frac{1}{l_{p}}=\frac{a_{\perp}^{3} / 2 V_{p}+\alpha_{p} a_{\perp}+6|\zeta(-1 / 2)|}{3 a_{\perp}}=0 .
$$

Since $\alpha_{p} a_{\perp} \gg 6|\zeta(-1 / 2)|$ for the lattice depths $V_{L}$ we can achieve in this experiment, Eq. S1 can be approximated using $1 / V_{p}=-2 \alpha_{p} / a_{\perp}^{2}$. By Taylor expanding this around the $3 \mathrm{D}$ resonance field $B_{3 D}$ to first order

$$
\left.\frac{1}{V_{p}}\right|_{B=B_{1 D}}=\left.\frac{1}{V_{p}}\right|_{B=B_{3 D}}+\left.\frac{\partial\left(1 / V_{p}\right)}{\partial B}\right|_{B=B_{3 D}}\left(B_{1 D}-B_{3 D}\right)=-\frac{2 \alpha_{p}}{a_{\perp}^{2}},
$$

we obtain a simple analytical form for the confinement-induecd shift $\delta_{B}\left(V_{L}, \alpha_{p}\right)=B_{1 D}-B_{3 D}$

$$
\begin{aligned}
\delta_{B} & =\frac{-2 \alpha_{p}}{\left.\frac{\partial\left(1 / V_{p}\right)}{\partial B}\right|_{B=B_{3 D} a_{\perp}^{2}}} \\
& =\frac{-2 m E_{r}}{\left.\hbar^{2} \frac{\partial\left(1 / V_{p}\right)}{\partial B}\right|_{B=B_{3 D}}} \alpha_{p} \sqrt{V_{L}},
\end{aligned}
$$

where $\left.\frac{\partial\left(1 / V_{p}\right)}{\partial B}\right|_{B=B_{3 D}}<0$.

\section{CONFINEMENT-INDUCED SHIFT $\delta_{B, 2 D}$ IN QUASI-2D}

Similarly to the confinement-induced shift in quasi-1D, an equivalent expression to Eq. S3 can be derived for this geometry by considering the quasi-2D scattering parameters [3]. The confinement-induced shift in quasi-2D $\delta_{B, 2 D}=B_{2 D}-B_{3 D}$ can be approximated by

$$
\delta_{B, 2 D}=\frac{1}{2} \delta_{B} .
$$

The open circles in Fig. S1 show the data of $\delta_{B}$ in quasi-2D from [4], and the solid curve shows the result of a fit to Eq. S4 with the effective range $\alpha_{p}=0.158(5) a_{0}^{-1}$ as a fitting parameter. This value of $\alpha_{p}$ is within $15 \%$ of the value the authors of [4] obtained by fitting measurements of the dissociation energy, as well as the value extracted from the fit to our quasi-1D data shown in the main text.

*randy@rice.edu 


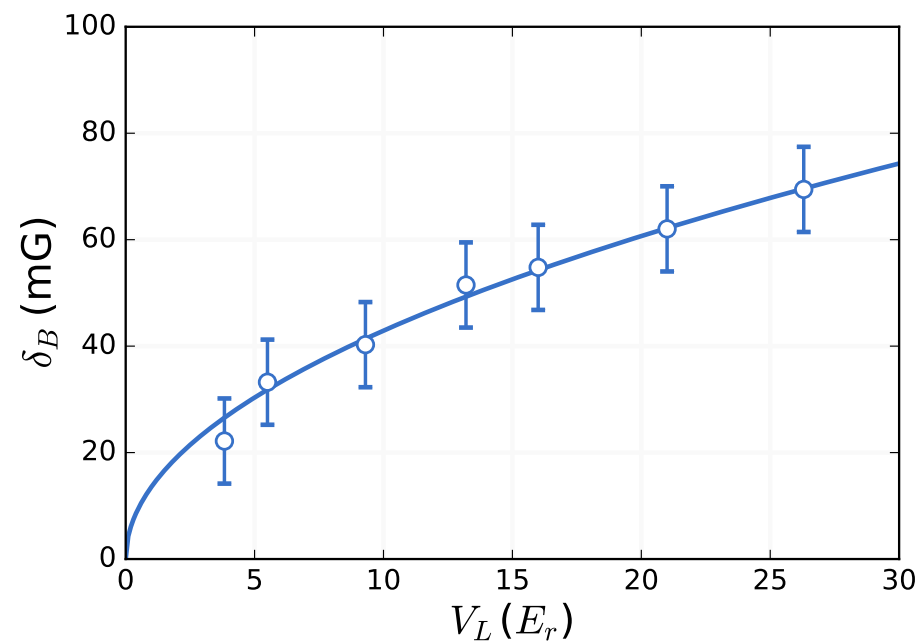

Figure S1. The open circles show the confinement-induced shift for the quasi-2D $p$-wave Feshbach resonance position as a function of the corresponding lattice depth (data from [4]). The solid curve shows the result of fit to Eq. S4, with the effective range $\alpha_{p}=0.158(5) a_{0}^{-1}$.

\section{THREE-BODY LOSS COEFFICIENT $L_{3}$ VERSUS $V_{L}$}

We measure the time evolution of atom number $N$ with $V_{L}$ between 15 and $75 E_{r}$ and extract $L_{3}$ by fitting to Eq. 4 in the main text. The peak $L_{3}$ as a function of $V_{L}$ is shown in Fig. S2.

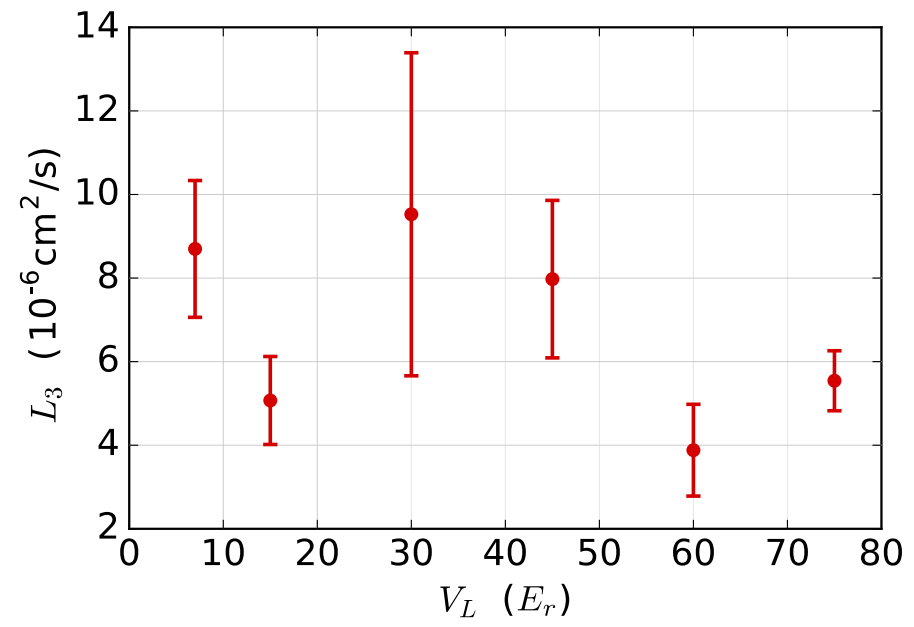

Figure S2. Peak three-body loss coefficient $L_{3}$ as a function of lattice depth $V_{L} . L_{3}$ is extracted by fitting the time evolution of $N$ to Eq. 4 as described in the Fig. 2(a) caption. Error bars indicate the one-sigma confidence interval for the fitting parameters $L_{3}$. 


\section{ANALYSIS USING IN SITU IMAGING WITH INVERSE ABEL TRANSFORM}

We probe atoms using in situ imaging and perform the inverse Abel transform on the column density, assuming cylindrical symmetry, to obtain the distribution of the number of atoms per tube $N_{t}(r)$. We group the tubes into separate cylindrical shells as shown in Fig. S3.
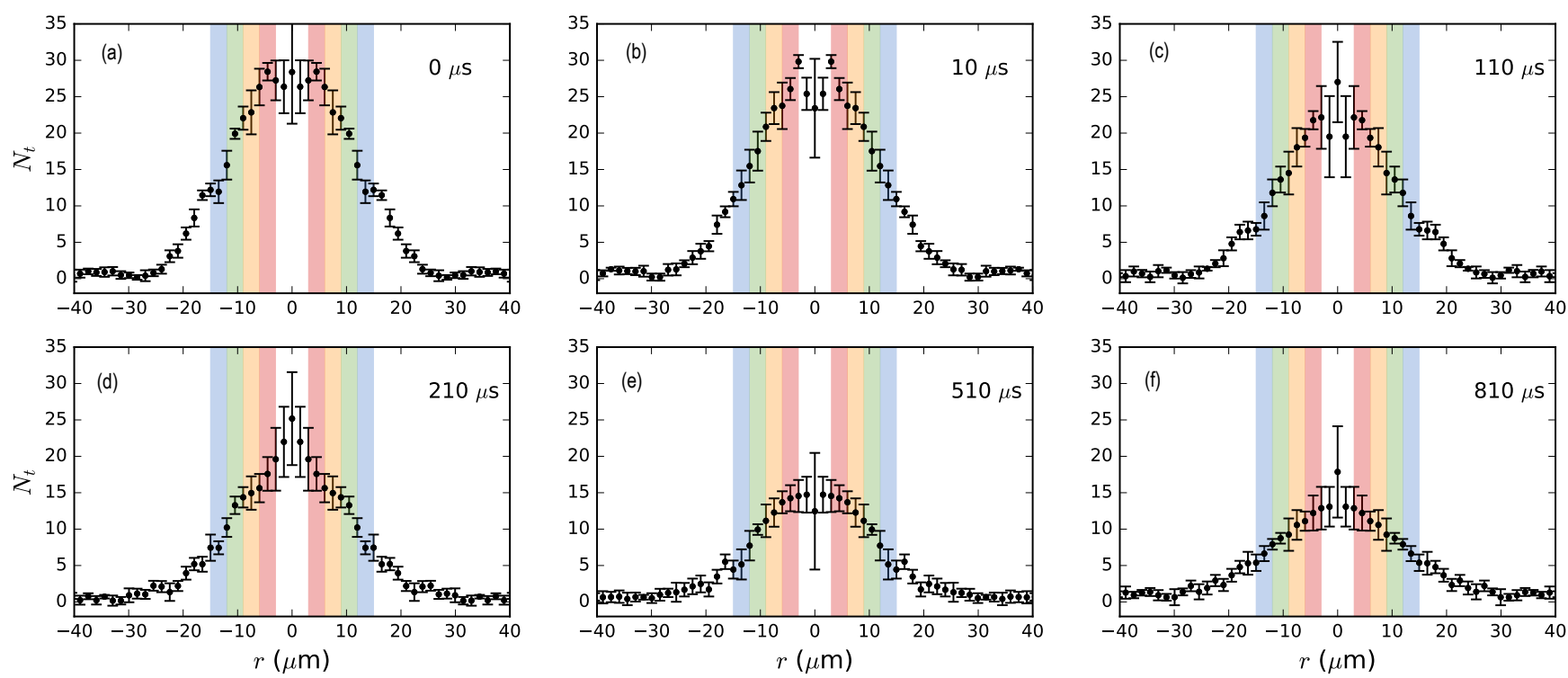

Figure S3. Typical time evolution of $N_{t}$ in a tube at a distance $r$ from the center of the 2D lattice. Regions with colored backgrounds correspond to the shells $i=1-4$ in the main text Fig. 2(b). Data points are averaged over 5 shots, and error bars indicate the standard error of the mean.

\section{TYPICAL TIME EVOLUTION OF $\left\langle N_{t}\right\rangle$ FITS TO THE CASCADE MODEL}

We extract $K_{a d}$ from the fits to Eq. 5 in the main text, using theoretical values of $K_{a a}$ and $\Gamma$ for $T=0.1 T_{F, 1}$. A typical time evolution with the fitted curve is shown in Fig. S4.

\section{QUASI-1D $p$-WAVE SCATTERING CROSS-SECTION}

The quasi-1D $p$-wave scattering amplitude is [3]

$$
f_{1 \mathrm{D}}(k)=\frac{-i k}{1 / l_{p}+\xi_{p} k^{2}+i k}
$$

In $1 \mathrm{D}$, the equivalent of the scattering cross-section is simply the modulus of the scattering amplitude squared

$$
\sigma_{1 \mathrm{D}}(k)=\left|f_{1 D}(k)\right|^{2}=\frac{k^{2}}{k^{2}+\left(1 / l_{p}+\xi_{p} k^{2}\right)^{2}},
$$

which is bounded from above by 1 . Near a Feshbach resonance for $l_{p}<0$, this expression may be approximated by a 
Lorentzian in terms of the collision energy, $E=\hbar^{2} k^{2} / m$ as follows:

$$
\sigma_{1 \mathrm{D}}(E) \approx \frac{\left(\frac{\Gamma}{2}\right)^{2}}{\left(E-E_{\mathrm{res}}\right)^{2}+\left(\frac{\Gamma}{2}\right)^{2}} .
$$

Here, $E_{\text {res }}=-\hbar^{2} / l_{p} \xi_{p} m>0$ for $l_{p}<0$ is the above-threshold binding energy of the Feshbach molecule, and $\Gamma=$ $\left(\hbar / \xi_{p}\right) \sqrt{4 E_{\mathrm{res}} / m}$ is the width of the resonance [5].

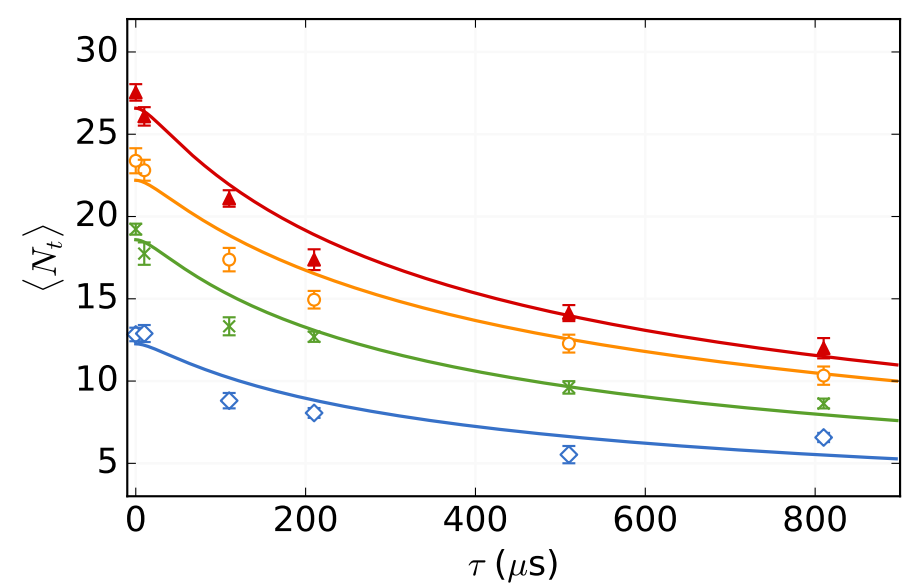

Figure S4. Typical time evolution of averaged tube population $\left\langle N_{t}\right\rangle$ in 4 shells at $\Delta B=30 \mathrm{mG}$ with $V_{L}=75 E_{r}$. Data are the same as Fig. 2(b) in the main text. Solid curves show fits to Eq. 5 in the main text with $K_{a a}$ and $\Gamma$ calculated for $T=0.1 T_{F, 1}$.

\section{PROBABILITY DENSITY FUNCTION OF $k_{r}$ FOR A TRAPPED FERMI GAS}

The probability density function of $k_{r}$ for a trapped Fermi gas is

$$
P\left(k_{r}\right)=\frac{\hbar}{N_{a}^{2}} \int_{-\infty}^{\infty} d k^{\prime} n\left(k^{\prime}\right) n\left(k^{\prime}-k_{r}\right),
$$

and the $k$-space number density is given by

$$
n(k)=\frac{1}{2 \pi \hbar} \int_{-\infty}^{\infty} d x \frac{1}{\exp \left[\beta\left(\frac{1}{2} m \omega_{\mathrm{z}}^{2} x^{2}+\frac{\hbar^{2} k^{2}}{2 m}-\mu\right)\right]+1},
$$

where $\beta=1 / k_{\mathrm{B}} T$ and $\mu$ is the chemical potential.

\section{DEPENDENCE OF $\kappa a_{\perp}$ ON $V_{L}$}

$\kappa$ is the magnitude of the wavevector related to the binding energy of the Feshbach molecule, which can be calculated by

$$
\kappa=\frac{\sqrt{m E_{\mathrm{res}}}}{\hbar}=\sqrt{\frac{-1}{l_{p} \xi_{p}}} .
$$


where $l_{p}$ and $\xi_{p}$ are the $1 \mathrm{D}$ scattering parameters modified from 3D scattering quantities $V_{p}$ and $\alpha_{p}$ with confinement strength $a_{\perp}$ as mentioned in the main text. By Taylor expanding $\kappa^{2}$ around the Feshbach resonance field $B_{1 \mathrm{D}}$ for a particular $V_{L}$, we find a constant $\kappa$ at a fixed magnetic field detuning $\Delta B$ which is independent of $V_{L}$ :

$$
\begin{aligned}
\kappa^{2}(\Delta B) & =\left.\kappa^{2}\right|_{B=B_{1 D}}+\left.\frac{\partial \kappa^{2}}{\partial B}\right|_{B=B_{1 D}} \Delta B+\left.\frac{\partial^{2} \kappa^{2}}{\partial B^{2}}\right|_{B=B_{1 D}} \Delta B^{2}+O\left(\Delta B^{3}\right) \\
& =\left.\frac{\partial\left(1 / V_{p}\right)}{\partial B}\right|_{B=B_{1 D}} \Delta B+\left.\frac{\partial^{2}\left(1 / V_{p}\right)}{\partial B^{2}}\right|_{B=B_{1 D}} \Delta B^{2}+O\left(\Delta B^{3}\right)
\end{aligned}
$$

Therefore, $\kappa a_{\perp}$ is proportional to $V_{L}^{1 / 4}$ for a particular $\Delta B$, as shown in Fig. S5.

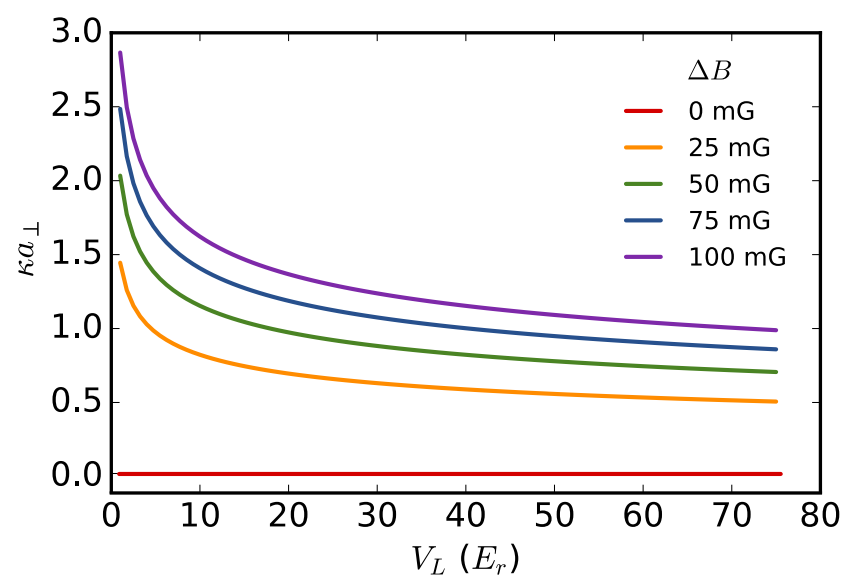

Figure S5. $\kappa a_{\perp}$ as a function of lattice depth $V_{L}$. Each curve is $V_{L}^{1 / 4}$ with a scaling factor set by $\Delta B$.

[1] B. E. Granger and D. Blume, Phys. Rev. Lett. 92, 133202 (2004).

[2] L. Pricoupenko, Phys. Rev. Lett. 100, 170404 (2008).

[3] D. V. Kurlov and G. V. Shlyapnikov, Phys. Rev. A 95, 032710 (2017).

[4] M. Waseem, Z. Zhang, J. Yoshida, K. Hattori, T. Saito, and T. Mukaiyama, J. Phys. B 49, 204001 (2016).

[5] V. Gurarie and L. Radzihovsky, Ann. Phys. (NY) 322, 2 (2007). 